\title{
Modulation of capecitabine administration to improve continuity of adjuvant chemotherapy for patients with colorectal cancer: A phase II study
}

\author{
YUKI MIZUMOTO $^{1}{ }^{,}$SHOZO YOKOYAMA ${ }^{1}$, KENJI MATSUDA ${ }^{1}$, HIROMITSU IWAMOTO ${ }^{1}$, \\ YASUYUKI MITANI ${ }^{1}$, KOICHI TAMURA ${ }^{1}$, YUKI NAKAMURA ${ }^{1}$, DAISUKE MURAKAMI ${ }^{1}$, \\ MASAMI OKA ${ }^{2}$, YASUHITO KOBAYASHI ${ }^{3}$ and HIROKI YAMAUE ${ }^{1}$
}

\author{
${ }^{1}$ Second Department of Surgery, School of Medicine, Wakayama Medical University, Wakayama 641-8510; \\ ${ }^{2}$ Department of Surgery, National Hospital Organization Minami Wakayama Medical Center, Wakayama 646-8558; \\ ${ }^{3}$ Department of Surgery, Wakayama Rosai Hospital, Wakayama 640-8505, Japan
}

Received January 26, 2019; Accepted October 8, 2019

DOI: $10.3892 / \mathrm{mco} .2019 .1961$

\begin{abstract}
Adjuvant chemotherapy with capecitabine is frequently not completed due to adverse events, including hand-foot syndrome. A higher completion rate of capecitabine by reduction of the side effects may improve disease-free survival and quality of life of affected patients. In the present study, colorectal cancer (CRC) patients were treated with capecitabine $\left(2,500 \mathrm{mg} / \mathrm{m}^{2} /\right.$ day $)$, which was taken for five days, followed by an interval of two days (5-days-on/2-days-off schedule). One course lasted three weeks, and eight courses (24 weeks) were administered. The median number of treatment courses was significantly higher in patients in the 5-days-on/2-days-off regimen group compared with that of patients in the retrospectively included conventional regimen group $(\mathrm{P}=0.0438)$. The frequency of completion of the scheduled treatment by patients in the 5-days-on/2-days-off regimen group was significantly higher $(\mathrm{P}=0.0389)$. The present phase II study suggests that toxicities associated with the 5-days-on/2-days-off regimen are lower compared with those of the conventional regimen, and that the occurrence of adverse events was higher, but less high-grade toxicities were reported. The time to treatment failure was also favorable in the new regimen and it demonstrated good feasibility. In conclusion, the present study demonstrated good feasibility with retained quality of life and acceptable adverse effects (mostly low-grade), and the 5-days-on/2-days-off regimen should be further evaluated in future randomized controlled trials. The present study was registered in the University
\end{abstract}

Correspondence to: Professor Hiroki Yamaue, Second Department of Surgery, School of Medicine, Wakayama Medical University, 811-1 Kimiidera, Wakayama 641-8510, Japan

E-mail: yamaue-h@wakayama-med.ac.jp

Key words: capecitabine, 5-days-on/2-days-off regimen, adjuvant chemotherapy, colorectal cancer, feasibility
Hospital Medical Information Network (UMIN) clinical trial registry (no. UMIN000012813).

\section{Introduction}

For colorectal cancer (CRC), 5-fluorouracil (5-FU) has been a major medicinal treatment. However, gastrointestinal adverse events and myelosuppression, are reportedly associated with the conventional 5-FU bolus schedule (1).

Capecitabine is an oral fluoropyrimidine that may replace 5-FU as a single-agent therapy for CRC due to its good efficacy, favorable tolerability and convenience. To compare capecitabine with bolus 5-FU/leucovorin (LV) therapy (Mayo Clinic regimen) as an adjuvant chemotherapy in stage III colon cancer, a phase III clinical study (X-ACT trial) has been performed (2-4). Capecitabine treatment was at least equivalent to 5-FU/LV therapy (Mayo Clinic regimen) regarding the primary endpoint of three-year disease-free survival (DFS) (5). Capecitabine also achieved improved relapse-free survival over 5-FU and was associated with significantly fewer adverse events (AEs) $(5,6)$.

However, AEs including hand-foot syndrome (HFS) still prevent patients from completing the adjuvant chemotherapy course. Twelves et al (5) reported that the grade $(\mathrm{G}) 3 / 4$ toxicity was $20 \%$ for hyperbilirubinemia, $17 \%$ for HFS and $11 \%$ for diarrhea (5). According to Emi et al (7), 24 out of 97 patients $(24.7 \%)$ discontinued the treatment due to side effects. The most common reason was HFS in 7 patients (7.2\%), followed by 5 patients with hematological toxicities $(5.2 \%)$ and 5 patients with liver dysfunction (5.2\%) (7). These adverse events prevent patients from completing the adjuvant chemotherapy courses. Overall DFS and quality of life (QOL) may be improved by a higher capecitabine completion rate, achieved by reduction of side effects.

Previous clinical studies have adopted the conventional oral capecitabine regimen, wherein the drug was taken for 14 consecutive days every 21 days. An alternative 5-days-on/2-days-off schedule of capecitabine has been proposed as a way to reduce side effects in patients with 
advanced solid cancers (8). According to this schedule, patients are treated with capecitabine $\left(2,500 \mathrm{mg} / \mathrm{m}^{2} /\right.$ day $)$, which is taken for five days, followed by an interval of two days. One course lasts for three weeks, and eight courses (24 weeks) are administered. Modulation of the methods of capecitabine administration may change the profile of the side effects of capecitabine and lead to an increased total dose and improved QOL.

The present study aimed to assess the potency of the 5-days-on/2-days-off schedule for patients with CRC compared with the conventional schedule for the capecitabine regimen.

\section{Patients and methods}

Inclusion criteria. The patients were enrolled between March 2014 and October 2014. The follow-up period was one year from the last case registration. Patients were enrolled at the Wakayama Medical University Hospital (Wakayama, Japan) and its associated teaching hospitals, National Hospital Organization Minami Wakayama Medical Center (Wakayama, Japan), Wakayama Rosai Hospital (Wakayama, Japan) and Izumiotsu Municipal Hospital (Osaka, Japan). Patients on the 5-days-on/2-days-off regimen who met all of the following criteria were eligible for the present study, regardless of sex: Histologically or cytologically diagnosed with CRC; capecitabine used as adjuvant chemotherapy; age between 20 and 80 years; Eastern Cooperative Oncology Group (ECOG) performance status (PS) of 0 or 1; principal organ functions sufficiently maintained (see criteria below); first-line treatment; written informed consent to participate in the study.

Furthermore, patients were required to be CRC stage III or high-risk stage II and undergoing curative operation followed by adjuvant chemotherapy (staging criteria in accordance with the TNM Classification of Malignant Tumors, 7th edition) (9). According to the American Society of Clinical Oncology guidelines (10) and the European Society for Medical Oncology guidelines (11), high-risk stage II CRC has $<12$ lymph nodes, T4 lesions, perforation, poorly differentiated histology, vascular or lymphatic or perineural invasion, and clinical presentation with intestinal occlusion or perforation.

The study was performed in accordance with the Declaration of Helsinki and the Ethical Guidelines for Clinical Research and has been approved by the Ethics Committee of Wakayama Medical University (Wakayama, Japan). Written informed consent was obtained from all patients prior to enrollment in this study.

Regarding the laboratory test results, the following criteria were also required to be satisfied: White cell count $>1,500 / \mathrm{mm}^{3}$; neutrophil count $\geq 1,500 / \mathrm{mm}^{3}$; platelet count $\geq 100,000 / \mathrm{mm}^{3}$; hemoglobin $\geq 9.0 \mathrm{~g} / \mathrm{dl}$; total bilirubin $\leq 1.5 \mathrm{mg} / \mathrm{dl}$; aspartate transaminase, alanine aminotransferase $\leq 100 \mathrm{IU} / 1$; serum creatinine $\leq 1.5 \mathrm{mg} / \mathrm{dl}$.

Exclusion criteria. Patients who met any of the following criteria were excluded from participation in the study: Pregnant or lactating; likely to, or planning to, become pregnant; history of malignancy; uncontrollable congestive heart failure, hypertension, angina pectoris, arrhythmia; severe drug hypersensitivity; side effects (or possible side effects) of fluoropyrimidine-type drugs with suspicion that dihydropyrimidine dehydrogenase deficiency may have developed; uncontrollable infection; oral ingestion difficulty; otherwise inappropriate for enrollment based on the researcher's judgment. Protocol treatment was not started in cases of rapid exacerbation after registration, protocol violation or discovery of ineligibility after registration.

Toxicities. Toxicities were graded according to the common terminology criteria for adverse events version 4.0 (12). Patients visited the hospital on the first day of admission and were checked for adverse events by blood examination and clinical examination. If a G3 adverse event was identified, the patient visited the clinic weekly until the adverse events were alleviated. If there were no G3 adverse events, the patient visited the clinic every three weeks. Adverse effects of capecitabine were checked by YM, SY, MO, YK and HY. If any discrepancies arose, all members discussed them in order to reach a consensus.

Treatment and assessment. The patients enrolled were treated with capecitabine $\left(2,500 \mathrm{mg} / \mathrm{m}^{2} / \mathrm{day}\right)$, which was taken on five consecutive days, followed by an interval of two days (5-days-on/2-days-off schedule). This trial was single-arm study and comparison was performed with a retrospectively enrolled conventional treatment group. The retrospective conventional treatment group was composed of 21 consecutive patients who received conventional treatment. They underwent surgery between January 2011 and March 2016 and received capecitabine treatment $\left(2,500 \mathrm{mg} / \mathrm{m}^{2} /\right.$ day $)$ taken on 14 consecutive days every 21 days according to the conventional schedule, prior to March 2014. One course lasted for three weeks, and eight courses ( 24 weeks) were administered in total. To assess the feasibility of the treatment schedule, sex, age, agreed date of acquisition of adverse events, ECOG PS, body height, body weight, body surface area, location of CRC, surgical procedure, lymph node dissection range, tumor diameter, histopathological classification, patient history, and comorbidities within one year were recorded. To examine hematologic side effects of the protocol treatment, baseline peripheral blood counts were measured and biochemical examination of blood was performed within 14 days prior to the start of drug administration.

General characteristics, clinical findings (subjective symptoms and objective signs), peripheral blood counts, blood chemistry values, tumor markers and incidence of HFS were typically recorded between weeks three and five during treatment. Imaging evaluation was performed at three months by computed tomography or magnetic resonance imaging. QOL evaluation (EORTC QLQ-C30) $(13,14)$ was performed prior to chemotherapy and after two, four, six and eight courses of treatment.

Treatment completion was defined as the completion of the eight three-week cycles on the 5-days-on/2-days-off schedule according to protocol in patients who were registered and began treatment within eight weeks post-operatively. None of the patients received neo-adjuvant chemotherapy.

Protocol discontinuation criteria. Discontinuation meant that the intake of the drug was completely terminated. Treatment was stopped for any of the following reasons: i) Decision 
made by the clinician to terminate protocol treatment due to AEs; ii) patient decided to discontinue treatment according to the protocol for reasons associated with AEs; iii) patient decided to stop treatment according to the protocol due to reasons not associated with AEs; iv) mortality during treatment.

Statistical analysis. Statistical analysis regarding patient characteristics was performed using non-parametric methods, including the Chi-squared test and Fisher's exact probability test. Statistical analysis regarding the treatment course, completion rate and relative dose intensity was performed using Fisher's exact probability test. For the time to treatment failure (TTF), evaluation was performed using Kaplan-Meier analysis and the log-rank test was used to identify statistically significant differences between the groups. The Cox proportional hazard ratios for the risk of treatment failure were calculated with a $95 \%$ confidence interval $(\mathrm{CI}) . \mathrm{P}<0.05$ was considered to indicate statistical significance.

\section{Results}

Patient characteristics. A total of 27 patients [18 males and 9 females; median age, 67 years (range 47-80 years)] were enrolled. There was no significant difference in the age and sex ratio of patients between the 5-days-on/2-days-off regimen group and the retrospectively included conventional regimen group. The follow-up period was one year from the last case registration. These patients underwent curative surgery and were diagnosed either as pathological stage III or high-risk stage II CRC. A total of 6 patients were excluded: One was changed to a different chemotherapy, one declined to participate in the trial, two patients started treatment from a dose lower than that prescribed in the study protocol, one was excluded due to bowel-diverting stoma operation and one lost the medicine after registration. A total of 21 patients were therefore eligible and treated with capecitabine $\left(2,500 \mathrm{mg} / \mathrm{m}^{2} /\right.$ day $)$, which was taken for five days followed by an interval of two days (5-days-on/2-days-off schedule). One course lasted for three weeks, and eight courses ( 24 weeks) were administered. The median age of patients on the 5-days-on/2-days-off schedule was 67 years (range, 38-80 years). The patients had a PS of 0 or 1 . Rectal cancer patients accounted for $23.8 \%$ (Table I). To compare the age, sex, PS, lymph node dissection, location of cancer, stage, invasion, lymphatic invasion, venous invasion and histological classification between the 5-days-on/2-days-off regimen group and conventional regimen group, two-sided $\mathrm{P}$-values were calculated and $\mathrm{P}<0.05$ was considered significant. A significant difference between the two groups was only identified in the stage (Table I).

Toxicities. The most common treatment-associated AEs were HFS (13 patients, 61.9\%), anemia (7 patients, 33.3\%), pigmentation (6 patients, $28.5 \%$ ), hyperbilirubinemia (6 patients, $28.5 \%$ ) and aspartate aminotransferase increase (6 patients, $28.5 \%$; Table II). The major G3/4 AE was HFS only (9.5\%). Although AEs in the conventional group were only investigated retrospectively, most common AEs were HFS (5 patients, 23.9\%) and hyperbilirubinemia (2 patients, 9.5\%). The AEs in the conventional group are provided in Table III. When comparing

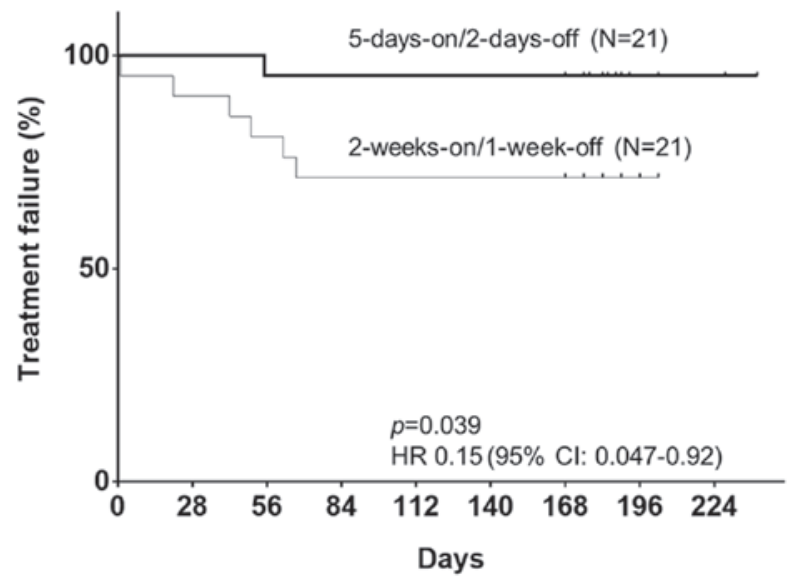

Figure 1. Kaplan-Meier plots of time to treatment failure of adjuvant chemotherapy with capecitabine for patients with stage III or high-risk stage II colorectal cancer after radical resection. Short vertical lines indicate censored data points. HR, hazard ratio.

the AEs between the two groups, it appeared that HFS was more common in the 5-days-on/2-days-off regimen but G3/4 AEs were less common.

Continuity of 5-days-on/2-days-off regimen as adjuvant chemotherapy for colorectal cancer. To address whether the 5-days-on/2-days-off regimen allows patients to continue adjuvant chemotherapy of stage III or high-risk stage II CRC, the median number and range of treatment courses in the 5-days-on/2-days-off regimen were compared with those of the conventional regimen. The median number of treatment courses in the 5-days-on/2-days-off regimen group was significantly higher than that in the conventional regimen group $(\mathrm{P}=0.0438)$. In terms of completion rate, 20 out of 21 patients $(95.2 \%)$ received the complete scheduled treatment according to the 5-days-on/2-days-off regimen. On the other hand, of the 21 patients following a conventional schedule, only 15 patients (71.4\%) completed the entire scheduled treatment (Table IV). Although the result was not significantly different $(\mathrm{P}=0.0931)$, the 5-days-on/2-days-off treatment tended to have better feasibility than the conventional regimen, and regarding the completion of the new vs. conventional regimen, the odds ratio was 8.0 (95\% CI: 0.87-73.7). When the TTF of the 5-days-on/2-days-off group was compared with that of the conventional group, the 5-days-on/2-days-off regimen group had a significantly lower risk of failure of the scheduled treatment $(\mathrm{P}=0.0389 ; \mathrm{HR}=0.15 ; 95 \%$ CI: 0.047-0.92; Fig. 1).

The median relative dose intensity was 1.0 (range, $0.15-1$ ) in the 5-days-on/2-days-off group and 1 (range, 0.125-1) in the conventional group, respectively. Table $\mathrm{V}$ presents the various reasons for discontinuation and dose reduction in the conventional group. A dose reduction was required for seven patients $(33.3 \%)$ in the 5-days-on/2-days-off group due to AEs. One of these seven patients discontinued the treatment. In the conventional group, four patients (19\%) reduced the capecitabine dose but completed all eight courses. The other six patients $(28.6 \%)$ stopped the regimen without dose reduction.

QOL of patients under the 5-days-on/2-days-off regimen. The results obtained with the EORTC QLQ-C30 are presented 
Table I. Patient characteristics.

\begin{tabular}{|c|c|c|c|}
\hline Characteristic & 5-days-on/2-days-off $(n=21)$ & Conventional $(n=21)$ & P-value \\
\hline Age (years) & $67(38-80)$ & $68(46-75)$ & 0.9354 \\
\hline Sex & & & 0.0614 \\
\hline Male & $6(28.6)$ & $12(57.1)$ & \\
\hline Female & $15(71.4)$ & $9(42.9)$ & \\
\hline PS & & & 1.0000 \\
\hline 0 & $21(100)$ & $21(100)$ & \\
\hline 1 & $0(0)$ & $0(0)$ & \\
\hline Lymph node dissection & & & 0.2931 \\
\hline D0 & $0(0)$ & $0(0)$ & \\
\hline D1 & $0(0)$ & $0(0)$ & \\
\hline $\mathrm{D} 2$ & $2(9.5)$ & $1(54.8)$ & \\
\hline D3 & $19(90.5)$ & $20(95.2)$ & \\
\hline Location of cancer & & & 0.9588 \\
\hline $\mathrm{C}$ & $1(4.8)$ & $2(9.5)$ & \\
\hline A & $4(19.0)$ & $4(19.0)$ & \\
\hline $\mathrm{T}$ & $2(9.5)$ & $3(14.3)$ & \\
\hline $\mathrm{D}$ & $1(4.8)$ & $1(4.8)$ & \\
\hline $\mathrm{S}$ & $8(38.1)$ & $6(28.6)$ & \\
\hline $\mathrm{RS}$ & $2(9.5)$ & $2(9.5)$ & \\
\hline $\mathrm{Ra}$ & $3(14.3)$ & $2(9.5)$ & \\
\hline $\mathrm{Rb}$ & $0(0)$ & $1(4.8)$ & \\
\hline Stage $^{a}$ & & & 0.0397 \\
\hline IIA & $5(23.8)$ & $0(0)$ & \\
\hline IIB & $1(4.8)$ & $0(0)$ & \\
\hline IIIA & $3(14.3)$ & $9(42.9)$ & \\
\hline IIIB & $12(57.1)$ & $11(52.4)$ & \\
\hline IIIC & $0(0)$ & $1(4.8)$ & \\
\hline Tumor depth of invasion & & & 0.0727 \\
\hline $\mathrm{T} 1$ & $2(9.5)$ & $4(19.0)$ & \\
\hline $\mathrm{T} 2$ & $1(4.8)$ & $5(23.8)$ & \\
\hline $\mathrm{T} 3$ & $17(81.0)$ & $8(38.1)$ & \\
\hline $\mathrm{T} 4$ & $1(4.8)$ & $4(19.0)$ & \\
\hline Lymphatic invasion & & & 0.6200 \\
\hline Ly0 & $11(52.4)$ & $10(47.6)$ & \\
\hline Ly1 & $6(28.6)$ & $6(28.6)$ & \\
\hline Ly2 & $3(14.3)$ & $5(23.8)$ & \\
\hline Ly3 & $1(4.8)$ & $0(0)$ & \\
\hline Venous invasion & & & 0.1885 \\
\hline V0 & $13(64.9)$ & $9(42.9)$ & \\
\hline V1 & $6(28.6)$ & $7(33.3)$ & \\
\hline $\mathrm{V} 2$ & $1(4.8)$ & $3(14.3)$ & \\
\hline V3 & $1(4.8)$ & $0(0.0)$ & \\
\hline Unknown & $0(0.0)$ & $2(9.5)$ & \\
\hline Histological classification & & & 0.4283 \\
\hline Pap & $1(4.8)$ & $0(0.0)$ & \\
\hline Wel & $7(33.3)$ & $10(47.6)$ & \\
\hline Mod & $13(61.9)$ & $11(52.4)$ & \\
\hline
\end{tabular}

${ }^{a}$ TNM classification. Tumor depth of invasion was defined as follows: T1, tumor invades submucosa; T2, tumor invades muscularis propria; T3, tumor invades through the muscularis propria into pericolorectal tissues; T4, tumor penetrates to the surface of the visceral peritoneum or tumor directly invades or is adherent to other organs or structures. Values are expressed as the median (range) or $\mathrm{n}(\%)$. PS, performance status; C, cecum; A, ascending colon; T, transverse colon; D, descending colon; S, sigmoid colon; Ra, upper rectum; Rb, lower rectum; Pap, papillary adenocarcinoma; Wel, well-differentiated adenocarcinoma; Mod, moderately differentiated adenocarcinoma. 
Table II. Adverse events in the 5-days-on/2-days-off regimen.

\begin{tabular}{|c|c|c|c|c|}
\hline Adverse event & All grades & Grade 1 & Grade 2 & Grade $3 / 4$ \\
\hline Diarrhea & $2(9.5)$ & $2(9.5)$ & $0(0.0)$ & $0(0.0)$ \\
\hline Hand-foot syndrome & $13(61.9)$ & $7(33.3)$ & $4(19.0)$ & $2(9.5)$ \\
\hline Nausea and vomiting & $1(4.7)$ & $1(4.7)$ & $0(0.0)$ & $0(0.0)$ \\
\hline Anorexia & $3(14.3)$ & $3(14.3)$ & $0(0.0)$ & $0(0.0)$ \\
\hline Dysgeusia & $3(14.3)$ & $2(9.5)$ & $1(4.7)$ & $0(0.0)$ \\
\hline Pigmentation & $6(28.5)$ & $6(28.5)$ & $0(0.0)$ & $0(0.0)$ \\
\hline Stomatitis & $2(9.5)$ & $2(9.5)$ & $0(0.0)$ & $0(0.0)$ \\
\hline Fatigue & $3(14.3)$ & $2(9.5)$ & $1(4.7)$ & $0(0.0)$ \\
\hline Neutropenia & $4(19.0)$ & $2(9.5)$ & $2(9.5)$ & $0(0.0)$ \\
\hline Anemia & $7(33.3)$ & $4(19.0)$ & $3(14.3)$ & $0(0.0)$ \\
\hline Thrombocytopenia & $1(4.7)$ & $1(4.7)$ & $0(0.0)$ & $0(0.0)$ \\
\hline Hyperbilirubinemia & $6(28.5)$ & $5(23.8)$ & $1(4.7)$ & $0(0.0)$ \\
\hline Alanine aminotransferase increase & $3(14.3)$ & $3(14.3)$ & $0(0.0)$ & $0(0.0)$ \\
\hline Aspartate aminotransferase increase & $6(28.5)$ & $6(28.5)$ & $0(0.0)$ & $0(0.0)$ \\
\hline
\end{tabular}

Values are expressed as $\mathrm{n}(\%)$.

Table III. Adverse events in the conventional regimen group.

\begin{tabular}{|c|c|c|c|c|}
\hline Adverse events & All grades & Grade 1 & Grade 2 & Grade 3/4 \\
\hline Diarrhea & $1(4.7)$ & $0(0.0)$ & $0(0.0)$ & $1(4.7)$ \\
\hline Hand-foot syndrome & $5(23.9)$ & $1(4.7)$ & $3(14.3)$ & $1(4.7)$ \\
\hline Anorexia & $1(4.7)$ & $0(0.0)$ & $0(0.0)$ & $1(4.7)$ \\
\hline Fatigue & $1(4.7)$ & $1(4.7)$ & $0(0.0)$ & $0(0.0)$ \\
\hline Hyperbilirubinemia & $2(9.5)$ & $0(0.0)$ & $2(9.5)$ & $0(0.0)$ \\
\hline Allergic reaction & $1(4.7)$ & $0(0.0)$ & $1(4.7)$ & $0(0.0)$ \\
\hline
\end{tabular}

Values are expressed as $\mathrm{n}(\%)$.

Table IV. Continuity in the 5-days-on/2-days-off regimen compared with the conventional regimen.

\begin{tabular}{|c|c|c|c|}
\hline Item & 5-days-on/2-days-off $(n=21)$ & Conventional $(\mathrm{n}=21)$ & P-value \\
\hline Treatment courses & $8.0(1-8)$ & $8.0(2-8)$ & 0.0438 \\
\hline Completion rate ${ }^{a}$ & & & 0.0931 \\
\hline Complete predetermined dose & $20(95.2)$ & $15(71.4)$ & \\
\hline Incomplete predetermined dose & $1(4.8)$ & $6(28.6)$ & \\
\hline Relative dose intensity & $1(0.15-1)$ & $1(0.125-1)$ & 0.158 \\
\hline
\end{tabular}

Values are expressed as median (range) or n (\%). ${ }^{\mathrm{a} O d d s}$ ratio for completion of the prescribed 5-days-on/2-days-off treatment vs. conventional treatment: $8.0 ; 95 \% \mathrm{CI}: 0.87-73.7$.

in Fig. 2. The QLQ-C30 questionnaire consists of two scales: A functional scale (Fig. 2A) and a symptom scale (Fig. 2B), each scoring from 0 to 100 . Higher scores are considered to indicate a better result on the functional scale and lower scores are better on the symptom scale. On the two scales, there was no significant change in any of the QOL functions and symptoms in the 5-days-on/2-days-off group over the course of the treatment $(\mathrm{P}>0.05)$.

Comparison of toxicities in the 5-days-on/2-days-off regimen with that in other studies. To address the degree of toxicity occurring under the 5-days-on/2-days-off regimen, the 
A Physical functioning

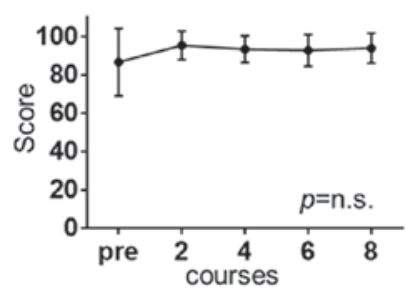

Emotional functioning
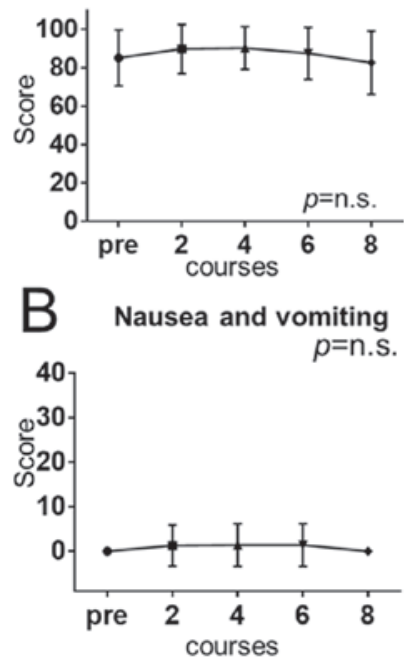

Pain

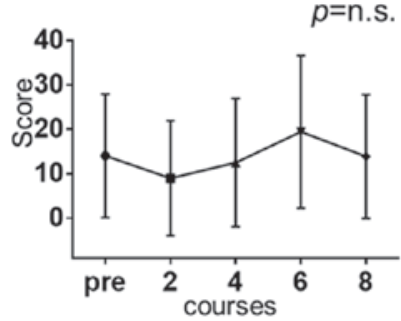

Constipation

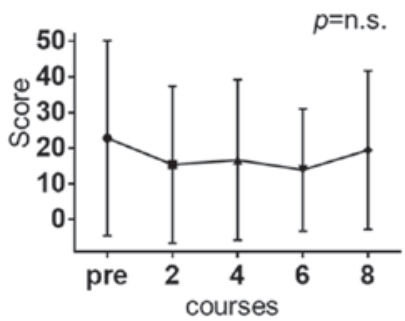

Role functioning

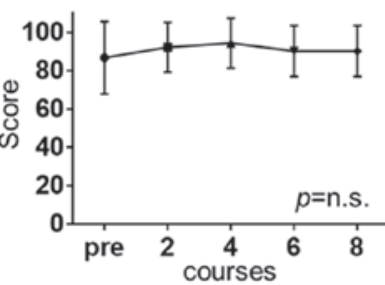

Social functioning

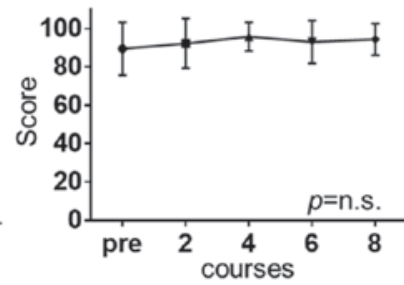

Fatigue

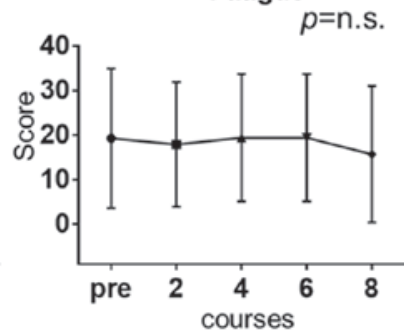

Insomnia

$p=$ n.s.

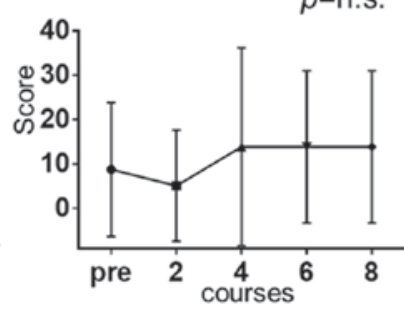

Diarrhea

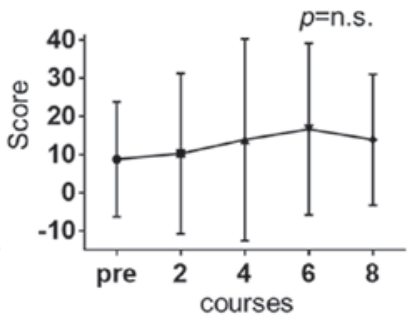

Cognitive functioning

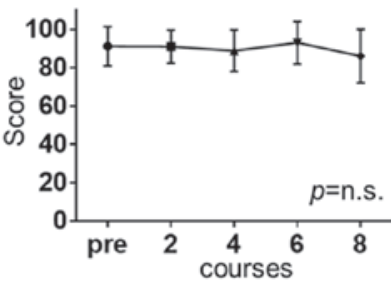

Global health status

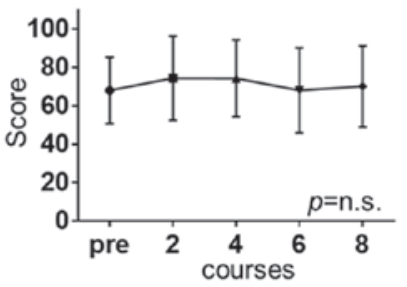

Dyspnea

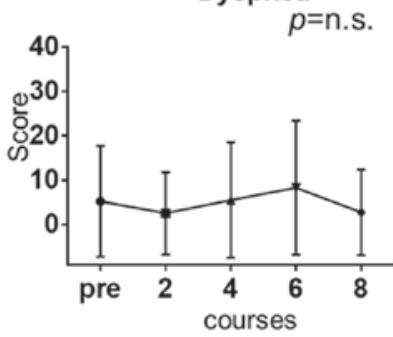

Appetite loss

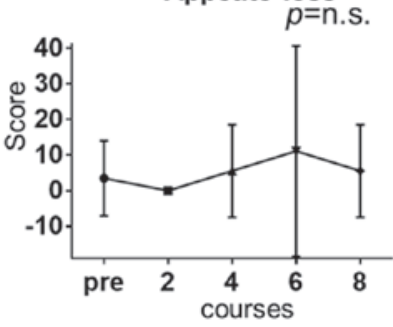

Financial difficulties

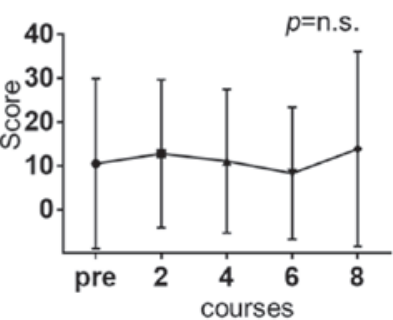

Figure 2. (A) Functional QOL scale for different courses of the 5-days-on/2-days-off regimen of capecitabine for adjuvant chemotherapy for CRC. (B) Symptom QOL scale for different courses of the 5-days-on/2-days-off regimen of capecitabine for CRC adjuvant chemotherapy. There was no significant difference from the score prior to treatment for any of the items. n.s., no significance; QOL, quality of life; CRC, colorectal cancer.

toxicities of the 5-days-on/2-days-off regimen were compared with those of other larger studies, including the Xeloda in Adjuvant Colon Cancer Therapy (X-ACT) (5) and Kyushu Study group of Clinical Cancer (KSCC)0803 (7) (Fig. 3). The X-ACT and KSCC0803 trials were performed using the 2-weeks-on/1-week-off regimen. Toxicities of all grades (Fig. 3A) and G3/4 toxicities (Fig. 3B) were compared among the three studies. The AEs in the present study, except for HFS, had a lower frequency and severity than those in the $\mathrm{X}$-ACT and KSCC0803 trials. In the present study, low-grade
HFS occurred at the same frequency but fewer cases of high-grade HFS were noted as compared with those in the $\mathrm{X}-\mathrm{ACT}$ and KSCC0803 trials.

\section{Discussion}

The conventional capecitabine regimen for adjuvant chemotherapy for stage III CRC patients, according to which the medicine is taken for 14 consecutive days every 21 days, has been reported to be equivalent to a bolus 5-FU/LV regimen 
Table V. Reasons for protocol dose reduction or incompletion.

\begin{tabular}{lcc}
\hline Item & $\begin{array}{c}\text { 5-days-on/ } \\
\text { 2-days-off }\end{array}$ & Conventional \\
\hline $\begin{array}{l}\text { Reason for dose reduction } \\
\text { HFS }\end{array}$ & 7 & 3 \\
Fatigue & 0 & 1 \\
Reason for incompletion & & \\
HFS & 1 & 1 \\
Total bilirubin increase & 0 & 2 \\
Anorexia & 0 & 1 \\
Rash & 0 & 1 \\
Diarrhea & 0 & 1 \\
\hline
\end{tabular}

HFS, hand-foot syndrome.
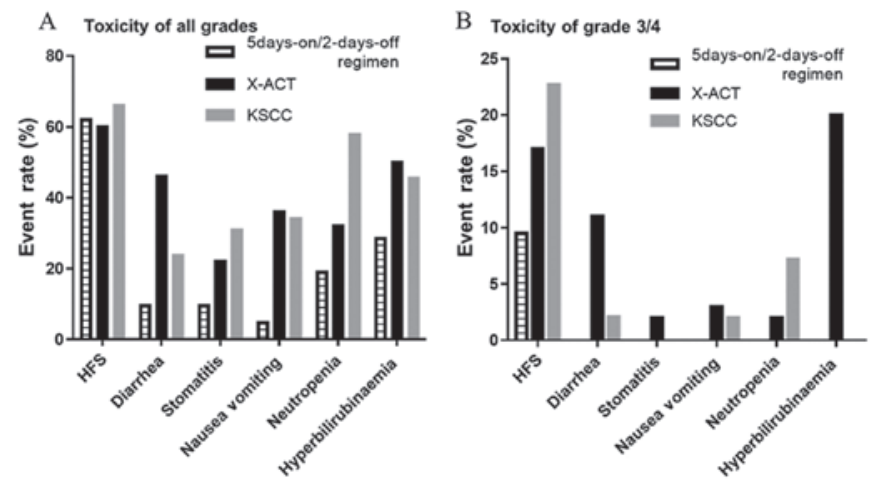

Figure 3. Comparison of toxicity among subjects of the X-ACT study, KSCC0803 study and 5-days-on/2-days-off regimen. (A) All grades of toxicity. (B) Grade 3/4 toxicities.

in a previous study (5). Capecitabine has been accepted as a standard adjuvant chemotherapy after radical surgery for patients with stage III CRC, but has considerable side effects. Twelves et al (5) reported an occurrence rate of all grades of HFS of $60 \%$, diarrhea of $46 \%$, nausea and vomiting of $36 \%$, stomatitis of $22 \%$, hyperbilirubinemia of $50 \%$ and neutropenia of $32 \%$. In a Japanese study on adjuvant capecitabine therapy for CRC, $66 \%$ of patients had symptoms of HFS, $23.7 \%$ of diarrhea, $34 \%$ of nausea and vomiting, $30.9 \%$ of stomatitis, $45.4 \%$ of hyperbilirubinemia and $57.7 \%$ of neutropenia. If these side effects are suppressed in patients undergoing adjuvant chemotherapy, their QOL may be improved and the regimen may be continued, leading to higher efficacy of adjuvant therapy.

In the present cohort under the 5-days-on/2-days-off regimen, diarrhea occurred in only $9.5 \%$ of patients and there were no G3 or G4 toxicities. The frequency of other side effects also tended to be lower. One reason for the 5-days-on/2-days-off regimen being more favorable than the 2-weeks-on/1-week-off regimen was provided by Pentheroudakis et al (8): Under the 5-days-on/2-days-off regimen, the time the drug prevails in the blood, presented by the maximum serum concentration $\mathrm{C}_{\max }$ or area under the curve for 5-FU, is extended as long as possible. Furthermore, it is desirable as an administration method, as no accumulation of the metabolite of 5-FU occurs (8). The emergence of AEs was then compared between the X-ACT study, the KSCC0803 study and the present 5-days-on/2-days-off regimen. All-grade toxicity of HFS was unmodulated by the change in administration method to the 5-days-on/2-days-off regimen. However, G3/4 toxicity of HFS and other toxicities were reduced under the 5-days-on/2-days-off regimen. It appears that the HFS was more common with the 5-days-on/2-days-off regimen but G3/4 AEs were less common. The median of the course during which HFS first occurred under the 5-day-on/2-day-off regimen was the third course, while it was the second course under the conventional regimen. There was no statistically significant difference regarding the median course of occurrence of HFS. $(\mathrm{P}<0.8411)$. Of note, only the patients in the experimental group (under the 5-day-on/2-day-off regimen) were enrolled in the present trial, and their behavior may have been influenced to continue the treatment, or the behavior of the doctors may have encouraged them to continue it. However, the patients in the retrospectively included conventional group did not participate in any clinical trial and no such bias existed for them. Therefore, the reason for the 5-day-on/2-day-off regimen having a better completion rate remains elusive, and a randomized controlled study is required to address this issue.

A limitation of the present study is that it is a single-arm preliminary study. The data of the group under the 5-days-on/2-days-off regimen were compared with those from external groups or the retrospective conventional 2-weeks-on/1-week-off regimen group. The present analysis included 21 patients in each cohort, and therefore, the sample size is not sufficiently large for detection of differences in efficacy between the two groups. Furthermore, although dose reduction under the 5-days-on/2-days-off regimen was precisely defined, there was no exact definition of dose reduction in the retrospectively included conventional regimen group. The comparison of regimen completion between the two groups is therefore limited. A randomized study is required to further assess the feasibility and safety of the 5-days-on/2-days-off regimen of capecitabine for adjuvant chemotherapy for CRC.

In spite of these limitations, the present study suggests that toxicities in the 5-days-on/2-days-off regimen were lower than in the conventional regimen. TTF was also favorable in the new regimen with a tendency toward good feasibility. The 5-days-on/2-days-off regimen of capecitabine may facilitate longer continuity of adjuvant chemotherapy for CRC patients. Although 5-FU monotherapy is the standard regimen for adjuvant chemotherapy, the combination of 5-FU with oxaliplatin is also an important modality of adjuvant chemotherapy for colon cancer. If a phase III randomized trial was to further demonstrate that a modified 5-days-on/2-days-off regimen improves the continuity of adjuvant chemotherapy, combination therapy of 5-FU with oxaliplatin should be evaluated in the next step.

\section{Acknowledgements}

The authors acknowledge the proofreading of the manuscript by Mr. Benjamin Phillis at the Clinical Study Support Center, Wakayama Medical University (Wakayama, Japan). 


\section{Funding}

No funding was received.

\section{Availability of data and materials}

All data generated or analyzed during this study are included in this published article.

\section{Authors' contributions}

YM and SY were responsible for the design and implementation of the study. YM performed the statistical analysis and drafted the manuscript. HY made substantial contributions to the conception, framework and design of the study. KM, KT, YM, HI, YN, DM, MO and YK made substantial contributions to clinical data collection and clinical management. All authors were involved in drafting, reading and approving the manuscript, and all agree to be accountable for the results. All authors read and approved the final manuscript.

\section{Ethics approval and consent to participate}

The study was performed in accordance with the Declaration of Helsinki and the Ethical Guidelines for Clinical Research and has been approved by the Ethics Committee of Wakayama Medical University (Wakayama, Japan). Written informed consent was obtained from all patients prior to enrollment in this study.

\section{Patient consent for publication}

Not applicable.

\section{Competing interests}

The authors declare that they have no competing interests.

\section{References}

1. Cassidy J, Twelves C, Van Cutsem E, Hoff P, Bajetta E, Boyer M, Bugat R, Burger U, Garin A, Graeven U, et al: First-line oral capecitabine therapy in metastatic colorectal cancer: A favorable safety profile compared with intravenous 5-fluorouracil/leucovorin. Ann Oncol 13: 566-575, 2002.

2. Reddy GK: Efficacy of adjuvant capecitabine compared with bolus 5-fluorouracil/leucovorin regimen in Dukes C colon cancer: Results from the X-ACT trial. Clin Colorectal Cancer 4 87-88, 2004.
3. Cassidy J, Douillard JY, Twelves C, McKendrick JJ, Scheithauer W, Bustova I, Johnston PG, Lesniewski-Kmak K, Jelic S, Fountzilas G, et al: Pharmacoeconomic analysis of adjuvant oral capecitabine vs intravenous 5-FU/LV in Dukes' $\mathrm{C}$ colon cancer: The X-ACT trial. Br J Cancer 94: 1122-1129, 2006.

4. Twelves C, Scheithauer W, McKendrick J, Seitz JF, Van Hazel G, Wong A, Diaz-Rubio E, Gilberg F and Cassidy J: Capecitabine versus 5-fluorouracil/folinic acid as adjuvant therapy for stage III colon cancer: Final results from the X-ACT trial with analysis by age and preliminary evidence of a pharmacodynamic marker of efficacy. Ann Oncol 23: 1190-1197, 2012.

5. Twelves C, Wong A, Nowacki MP, Abt M, Burris H III, Carrato A, Cassidy J, Cervantes A, Fagerberg J, Georgoulias V, et al: Capecitabine as adjuvant treatment for stage III colon cancer. N Engl J Med 352: 2696-2704, 2005.

6. Twelves CJ: Xeloda in Adjuvant Colon Cancer Therapy (X-ACT) trial: Overview of efficacy, safety, and cost-effectiveness. Clin Colorectal Cancer 6: 278-287, 2006.

7. Emi Y, Kakeji Y, Oki E, Saeki H, Ando K, Kitazono M, Sakaguchi Y, Morita M, Samura H, Ogata Y, et al: Initial report of KSCC0803: Feasibility study of capecitabine as adjuvant chemotherapy for stage III colon cancer in Japanese patients. Int J Clin Oncol 18: 254-259, 2013.

8. Pentheroudakis G, Pappas P, Golfinopoulos V, Fountzilas G, Nikolaidou M, Boumba VA, Vougiouklakis T, Nikiforidis L, Tzamakou E, Siarabi O, et al: Weekday on-weekend off oral capecitabine: A phase I study of a continuous schedule better simulating protracted fluoropyrimidine therapy. Cancer Chemother Pharmacol 60: 733-739, 2007.

9. Sobin LH, Gospodarowicz MK and Wittekind C: TNM classification of malignant tumours. 7th Edition. Wiley-Blackwell, Chichester, 2009.

10. Benson AB III, Schrag D, Somerfield MR, Cohen AM, Figueredo AT, Flynn PJ, Krzyzanowska MK, Maroun J, McAllister P, Van Cutsem E, et al: American Society of Clinical Oncology recommendations on adjuvant chemotherapy for stage II colon cancer. J Clin Oncol 22: 3408-3419, 2004.

11. Schmoll HJ, Van Cutsem E, Stein A, Valentini V, Glimelius B, Haustermans K, Nordlinger B, van de Velde CJ, Balmana J, Regula J, et al: ESMO consensus guidelines for management of patients with colon and rectal cancer. A personalized approach to clinical decision making. Ann Oncol 23: 2479-2516, 2012.

12. National Cancer Institute (NCI): Common Terminology Criteria for Adverse Events (CTCAE). Version 4.0. http://ctep.cancer. gov/protocolDevelopment/electronic_applications/ctc.htm.

13. Aaronson NK, Ahmedzai S, Bergman B, Bullinger M, Cull A, Duez NJ, Filiberti A, Flechtner H, Fleishman SB and de Haes JC: The European Organization for Research and Treatment of Cancer QLQ-C30: A quality-of-life instrument for use in international clinical trials in oncology. J Natl Cancer Inst 85: 365-376, 1993 .

14. Kobayashi K, Takeda F, Teramukai S, Gotoh I, Sakai H, Yoneda S, Noguchi Y, Ogasawara H and Yoshida K: A cross-validation of the European Organization for Research and Treatment of Cancer QLQ-C30. Eur J Cancer 34: 810-815, 1998.

This work is licensed under a Creative Commons Attribution-NonCommercial-NoDerivatives 4.0 International (CC BY-NC-ND 4.0) License. 